\title{
Differential Regulation of c-di-GMP Metabolic Enzymes by Environmental Signals Modulates Biofilm Formation in Yersinia pestis
}

\author{
Gai-Xian Ren', Sai Fan², Xiao-Peng Guo', Shiyun Chen ${ }^{3}$ and Yi-Cheng Sun ${ }^{1 *}$ \\ ${ }^{1} \mathrm{MOH}$ Key Laboratory of Systems Biology of Pathogens, Institute of Pathogen Biology, Chinese Academy of Medical \\ Sciences and Peking Union Medical College, Beijing, China, ${ }^{2}$ Institute of Nutrition and Food Hygiene, Beijing Centre for \\ Disease Control and Prevention, Beijing, China, ${ }^{3}$ Key Laboratory of Special Pathogens and Biosafety, Center for Emerging \\ Infectious Diseases, Wuhan Institute of Virology, Chinese Academy of Sciences, Wuhan, China
}

\section{OPEN ACCESS}

Edited by:

Catherine Ayn Brissette University of North Dakota School of Medicine and Health Sciences,

USA

Reviewed by:

Haichun Gao,

Zhejiang University, China Yanping Han,

Beijing Institute of Microbiology and Epidemiology, China

*Correspondence: Yi-Cheng Sun

sunyc@ipbcams.ac.cn

Specialty section:

This article was submitted to Microbial Physiology and Metabolism, a section of the journa Frontiers in Microbiology

Received: 13 April 2016 Accepted: 16 May 2016 Published: 03 June 2016

Citation: Ren G-X, Fan S, Guo X-P, Chen S and Sun Y-C (2016) Differential Regulation of c-di-GMP Metabolic Enzymes by Environmental Signals Modulates Biofilm Formation in Yersinia pestis.

Front. Microbiol. 7:821. doi: 10.3389/fmicb.2016.00821
Cyclic diguanylate (c-di-GMP) is essential for Yersinia pestis biofilm formation, which is important for flea-borne blockage-dependent plague transmission. Two diguanylate cyclases (DGCs), HmsT and HmsD and one phosphodiesterase (PDE), HmsP are responsible for the synthesis and degradation of $\mathrm{c}$-di-GMP in Y. pestis. Here, we systematically analyzed the effect of various environmental signals on regulation of the biofilm phenotype, the c-di-GMP levels, and expression of HmsT, HmsD, and HmsP in $Y$. pestis. Biofilm formation was higher in the presence of non-lethal high concentration of $\mathrm{CaCl}_{2}, \mathrm{MgCl}_{2}, \mathrm{CuSO}_{4}$, sucrose, sodium dodecyl sulfate, or dithiothreitol, and was lower in the presence of $\mathrm{FeCl}_{2}$ or $\mathrm{NaCl}$. In addition, we found that $\mathrm{HmsD}$ plays a major role in biofilm formation in acidic or redox environments. These environmental signals differentially regulated expression of $\mathrm{HmsT}, \mathrm{HmsP}$ and $\mathrm{HmsD}$, resulting in changes in the intracellular levels of $\mathrm{C}$-di-GMP in $Y$. pestis. Our results suggest that bacteria can sense various environmental signals, and differentially regulate activity of DGCs and PDEs to coordinately regulate and adapt metabolism of c-di-GMP and biofilm formation to changing environments.

Keywords: environmental signals, biofilm, c-di-GMP, diguanylate cyclases, phosphodiesterase, Yersinia pestis

\section{INTRODUCTION}

Yersinia pestis, the causative agent of plague, is a unique Gram-negative bacterium that adopts an arthropod-borne route of transmission. Y. pestis maintains its existence by a cycle involving two hosts: a mammal (usually a rodent) and an insect (a flea). In the flea host, the bacteria can form a biofilm in the proventriculus (a valve between the midgut and esophagus), which interferes with and can eventually block the ingestion of blood (Hinnebusch et al., 1996; Jarrett et al., 2004; Hinnebusch and Erickson, 2008). As a result of this blockage-induced starvation, fleas with complete or partial blockage will repeatedly try to feed on hosts, resulting in regurgitation of ingested blood and bacteria into the bite site to infect the mammal (Bacot and Martin, 1914; Bacot, 1915). Y. pestis biofilm formation requires the hmsHFRS operon, which is responsible for biosynthesis of a polymeric $\beta-1,6-N$-acetyl-D-glucosamine-containing extracellular polysaccharide (EPS; Hinnebusch et al., 1996; Darby, 2008). 
As in many other bacteria, production of EPS in $Y$. pestis is dependent on the intracellular levels of cyclic diguanylate (c-di-GMP; Sun et al., 2011; Bobrov et al., 2014). c-di-GMP, an intracellular second messenger molecule, controls biofilm formation, EPS production, motility, virulence, and many other cellular processes (Hengge, 2009; Romling, 2012; Romling et al., 2013). c-di-GMP is synthesized by diguanylate cyclases (DGCs) that contain a GGDEF domain, and is hydrolyzed by phosphodiesterases (PDEs) that contain an EAL or HDGYP domain (Ryjenkov et al., 2005; Schmidt et al., 2005; Ryan et al., 2006). Bacteria are constantly exposed to changing environments, requiring that they coordinately regulate DGCs and PDEs levels to adjust the intracellular concentration of c-di-GMP to changes in the environment. Some environmental signals, such as temperature, light, oxygen, amino acids, growth medium and growth conditions, regulate c-di-GMP level and biofilm formation (Bernier et al., 2011; Malone et al., 2012; Koestler and Waters, 2013, 2014; Townsley and Yildiz, 2015). c-di-GMP signaling networks usually contain a large number of DGCs and PDEs that contribute to changes in the intracellular concentration of c-di-GMP. This redundancy suggests that the intracellular level of c-di-GMP reflects the cumulative activities of a complex composite of different DGCs and PDEs. Thus it is challenging to study how the concentration of c-di-GMP is modulated by environmental conditions.

Unlike other bacteria, the $Y$. pestis genome only contains three genes encoding enzymatically functional DGCs and PDEs (Bobrov et al., 2011; Sun et al., 2011). hmsT and hmsD, which encode DGCs, are responsible for synthesis of c-di-GMP, while $h m s P$, which encodes a PDE, is responsible for degradation of c-di-GMP (Kirillina et al., 2004; Bobrov et al., 2011; Sun et al., 2011).

$h m s D$ is a member of the $h m s C D E$ operon and is transcribed by the $h m s C$ promoter (Sun et al., 2011). HmsC, a periplasmic protein, inhibits inner membrane protein HmsD DGC activity (Bobrov et al., 2014; Ren et al., 2014), while the outer membrane protein HmsE counteracts with $\mathrm{HmsC}$ to activate $\mathrm{HmsD}$ (Bobrov et al., 2014). HmsT plays a dominant role in biofilm formation in vitro, while $\mathrm{HmsD}$ plays a major role in producing proventricular-blocking biofilm during flea colonization (Sun et al., 2011). Hfq, a small RNA chaperone involved in regulation of HmsT and HmsP expression (Bellows et al., 2012), regulates biofilm formation in the flea and in vitro (Bellows et al., 2012; Rempe et al., 2012). Recently we found that $3^{\prime}$ untranslated region ( $3^{\prime}$ UTR) negatively regulates the $h m s T$ expression in response to high temperature, allowing $Y$. pestis to adjust biofilm formation to the temperature of the host environment (Zhu et al., 2016). Although, only three enzymes are involved in maintaining c-diGMP homeostasis in $Y$. pestis, the environment signals and the mechanisms by which Hms T, HmsD and HmsP, are regulated to control biofilm formation are not well-studied. The only known environmental signal that affects the expression of these enzymes in $Y$. pestis is temperature: Hms is highly expressed at $26^{\circ} \mathrm{C}$ but not at $37^{\circ} \mathrm{C}$ (Perry et al., 2004; Zhu et al., 2016).

Little is known about environmental conditions in the digestive tract of the flea (Perry and Fetherston, 1997). In this study, we chose 12 different environmental conditions in vitro to simulate the environmental conditions in the gut of flea. We evaluated the effects of these environmental conditions in vitro on regulation of the biofilm phenotype, the c-di-GMP level, and the expression of HmsT, HmsCDE, and HmsP in Y.pestis. We found that changes in the environment affected the regulation of HmsT, HmsCDE, and HmsP, resulting in altered levels of intracellular c-di-GMP and differences in biofilm formation. Our findings may help to understand the regulatory mechanism of c-di-GMP signaling and biofilm formation in $Y$. pestis and in other bacteria.

\section{RESULTS}

\section{Y. pestis Biofilm Formation in Various Environments}

Biofilm formation might be regulated by different environmental signals. These signals might include the changes in $\mathrm{pH}$, redox state, envelope stress, metal ions, and osmolarity and as well as other parameters. To determine the effect of different environmental signals on biofilm formation, we investigated the effect of 12 different environmental conditions on the growth and biofilm formation of $Y$. pestis KIM6+ in vitro (Figure 1 and Supplementary Figure S1). Consistent with previous findings, biofilm formation of $Y$. pestis KIM6+ decreased as the temperature increased (Figure 1A). Addition of a low concentration of dithiothreitol (DTT; 1 or $2 \mathrm{mM}$ ) did not affect growth but decreased biofilm formation, while addition of $4 \mathrm{mM}$ DTT resulted in slow growth but increased biofilm formation (Figure 1B). Biofilm formation was increased by addition of moderate concentration of sucrose, $\mathrm{CuSO} 4, \mathrm{CaCl}_{2}$, $\mathrm{MgCl}_{2}$, or sodium dodecyl sulfate (SDS) were added in to the media (Figures 1C-G), while bacterial growth was not strongly affected by these compounds (Supplementary Figures S1C-G). Conversely biofilm formation (Figures 1C-G) and bacterial growth (Supplementary Figures S1C-G) were inhibited when high concentrations of these compounds were employed. Biofilm formation but not bacterial growth was inhibited by $\mathrm{FeCl}_{2}$ (Figure $\mathbf{1 H}$ and Supplementary Figure $\mathrm{S} 1 \mathrm{H}$ ). Addition of non-toxic concentrations of $\mathrm{H}_{2} \mathrm{O}_{2}$, or 2,2'-dipyridyl (DIP; an iron chelator) did not markedly affect $Y$. pestis KIM6+ biofilm formation (Figures 1I,J and Supplementary Figures S1I,J). A low pH strongly inhibited Y. pestis KIM6+ growth but did not markedly affect its biofilm formation, while a high $\mathrm{pH}$ strongly repressed biofilm formation and growth (Figure 1K and Supplementary Figure S1K). In addition, biofilm formation was inhibited by both low and high concentrations of $\mathrm{NaCl}$ (Figure 1L), while bacterial growth was only strongly inhibited by a high concentration of $\mathrm{NaCl}$ (Supplementary Figure S1L).

To identify the roles of HmsT and HmsD in different environmental conditions, we assessed the biofilm formation in the $h m s T$ and $h m s D$ mutants in various environments. Consistent with the previous findings that $h m s T$ plays a major role in biofilm formation in vitro (Bobrov et al., 2011; Sun et al., 2011), biofilm formation in the $h m s T$ mutant was undetectable under most of environmental conditions tested (Figure 1), except in acidic ( $\mathrm{pH}$ ) or redox (addition of $4 \mathrm{mM} \mathrm{DTT}$ ) environments (Figures 1B,K). On the other hand, biofilm formation was lower 
A

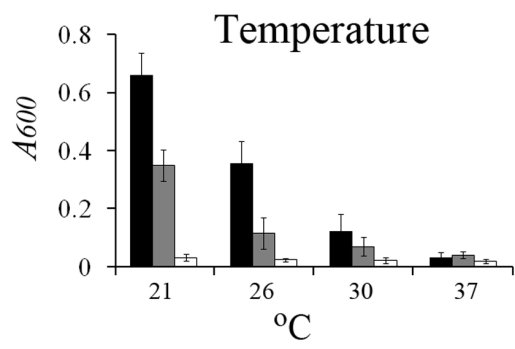

D

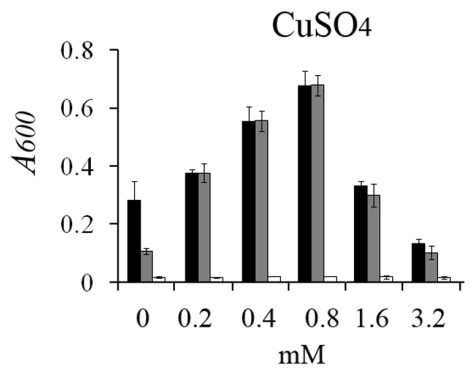

G

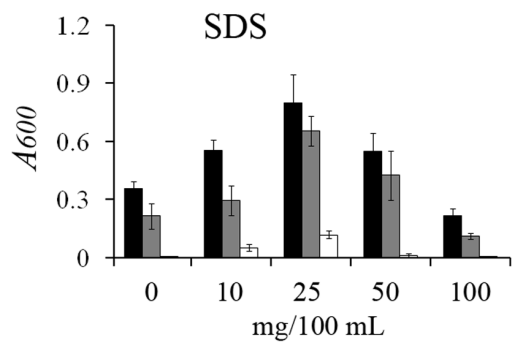

J

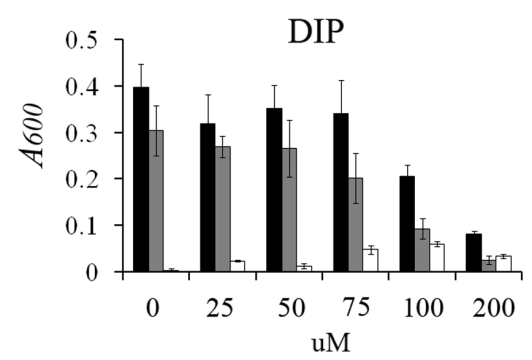

B

H

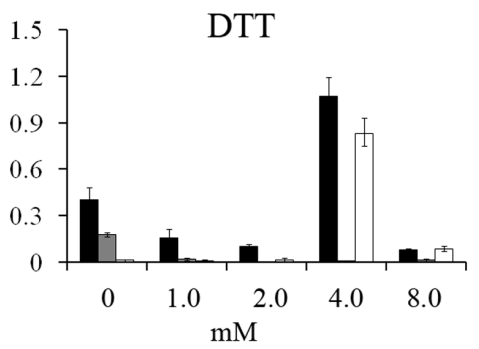

E

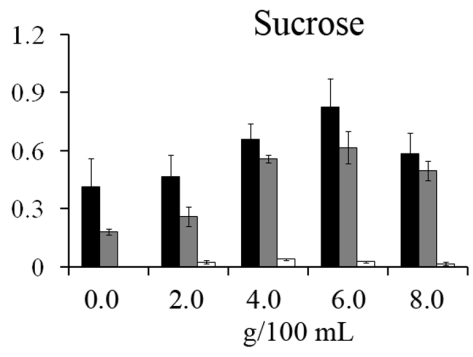

F
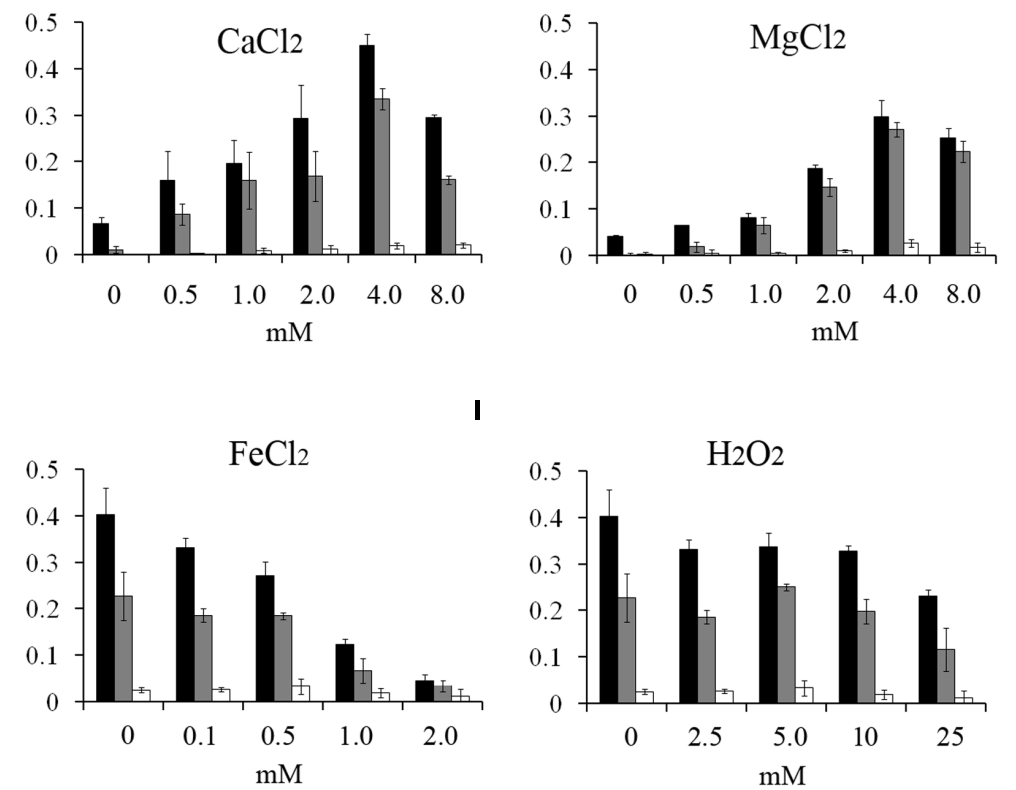

$\mathbf{L}$
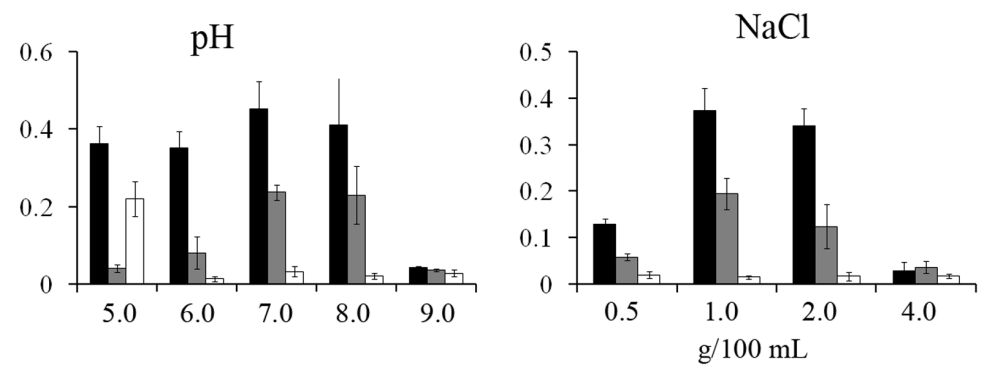

FIGURE 1 | Effect of environmental conditions on Yersinia pestis biofilm formation in vitro. Relative amounts of adherent biofilm formed by the $Y$. pestis. $\mathrm{KIM}$ + parental strain (black bars) and its isogenic derivatives, the hmsD mutant (gray bars) and the hmsT mutant (white bars), in various environmental conditions, (A, bacteria culture at different temperature, B-L, media supplemented with different concentrations of DTT, sucrose, $\mathrm{CuSO}_{4}, \mathrm{CaCl}_{2}, \mathrm{MgCl}_{2}, \mathrm{SDS}, \mathrm{FeCl}_{2}, \mathrm{DIP}, \mathrm{pH}$, $\mathrm{NaCl})$. The mean and standard deviation of three independent experiments are indicated.

in the $h m s D$ mutant in acidic ( $\mathrm{pH}$ 5) and redox (addition of $4 \mathrm{mM}$ DTT) environments (Figures 1B,K). These results suggest that $h m s D$ might be upregulated in acidic or redox environments, and that it might be important for biofilm formation in these two environments. Addition of SDS, a membrane-disrupting detergent, not only markedly increased biofilm formation in the wild-type and $h m s D$ mutant, but also resulted in detectable biofilm formation by the hmsT mutant (in the presence of 
$25 \mathrm{mg} / 100 \mathrm{~mL} \mathrm{SDS}$ ), indicating that SDS might not affect biofilm formation via the regulation of $h m s T$ or $h m s D$, but via the regulation of $h m s P$ or other unknown biofilm related genes (Figure 1G). Taken together, these results suggest that differential expression of $h m s T$, $h m s P$, and $h m s D$ expression might occur in response to various environmental signals and that these three genes might function together to regulate biofilm formation.

\section{The Intracellular c-di-GMP Levels of $Y$. pestis at Various Environments}

Because biofilm formation in $Y$. pestis is affected by the c-diGMP level (Kirillina et al., 2004; Simm et al., 2005; Bobrov et al., 2011; Sun et al., 2011), we reasoned that the effects of environmental conditions on biofilm formation might be due to changes in the cellular c-di-GMP level. Thus, we determined the intracellular c-di-GMP levels of $Y$. pestis under various environment conditions ( $4 \mathrm{mM} \mathrm{CaCl}, 4 \mathrm{mM} \mathrm{MgCl}_{2}, 2 \mathrm{mM}$ $\mathrm{FeCl}_{2}, 100 \mu \mathrm{M}$ DIP, $1 \mathrm{mM} \mathrm{CuSO} 4,0.01 \%$ SDS, 6\% sucrose, $4 \mathrm{mM}$ DTT, $10 \mathrm{mM} \mathrm{H}_{2} \mathrm{O}_{2}$, or $4 \% \mathrm{NaCl}$, or modified to $\mathrm{pH}$, and at high and low growth temperatures) that did not severely affect bacteria growth (Supplementary Figure S1). Consistent with the biofilm assay, the c-di-GMP level was increased by sucrose, $\mathrm{CuSO}_{4}$, $\mathrm{CaCl}_{2}$, SDS or DTT, decreased by $\mathrm{FeCl}_{2}$ or $\mathrm{NaCl}$, and not affected by $\mathrm{H}_{2} \mathrm{O}_{2}$ or DIP (Figure 2). High concentration of $\mathrm{FeCl}_{2}$ might inhibited the function of Fur, the global ferric uptake regulator, that has been reported regulate $h m s T$ expression, c-di-GMP level and biofilm formation in Y. pestis (Sun F. et al., 2012). Although, biofilm formation was increased by $\mathrm{MgCl}_{2}$ and decreased with temperature increase, the c-di-GMP levels were not significantly changed (Figure 2), suggesting that this environmental signal affects other unknown factors that regulate biofilm formation. It has been reported the hmsHFRS operon is downregulated by hyperosmotic stress (Han et al., 2005). Taken together these results suggested that many environmental signals affect the intracellular levels of c-di-GMP, which in turn, regulates biofilm formation in $Y$. pestis.

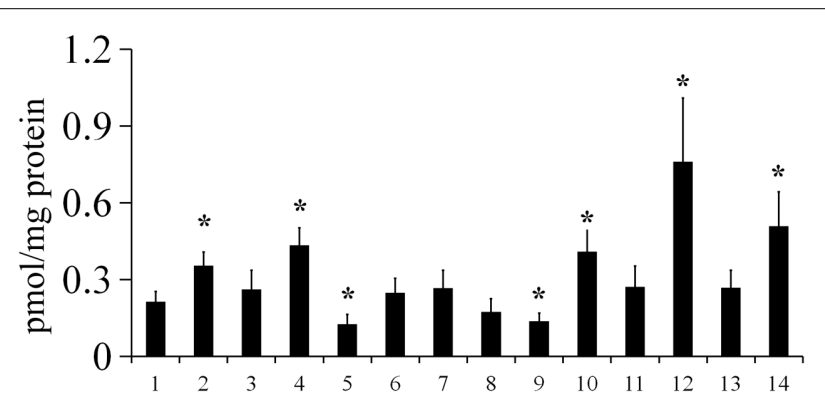

FIGURE 2 | The intracellular c-di-GMP level in $Y$. pestis in various environmental conditions. C-di-GMP was extracted from $Y$. pestis grown in LB medium at $26^{\circ} \mathrm{C}(1), 21^{\circ} \mathrm{C}(7)$, or $37^{\circ} \mathrm{C}(8)$, or in LB medium supplemented with $\mathrm{CaCl}_{2}$ (2), $\mathrm{MgCl}_{2}$ (3), $\mathrm{CuSO}_{4}$ (4), $\mathrm{FeCl}_{2}$ (5), $\mathrm{NaCl}$ (9), sucrose (10), $\mathrm{H}_{2} \mathrm{O}_{2}$ (11), DTT (12), DIP (13), or SDS (14), or at pH 5 (6) at $26^{\circ} \mathrm{C}$, and measured as described in the Experimental Procedures. ${ }^{*} P<0.05$. The mean and standard deviation of three independent experiments are indicated.

\section{Expression of PDE and DGCs at Different Growth Phase}

Because DGCs and PDEs expression might be affected by growth phase, we determined $h m s T, h m s C D E$, and $h m s P$ expression at the transcriptional and protein levels at different growth phases. To examine transcriptional expression of $h m s T, h m s C D E$ and $h m s P$, we deleted the $Y$. pestis lac $Z$ gene and constructed transcriptional fusions using Escherichia coli lac $Z$ as the reporter as previously described (Sun Y.C. et al., 2012). All fusions were integrated into the chromosome at their native locus. Bacteria were grown in broth to early exponential $\left(\mathrm{EE}, \mathrm{OD}_{600}\right.$ 0.4-0.6), mid-exponential ( $\mathrm{ME}, \mathrm{OD}_{600} 1.0-1.2$ ), early stationary (ES, $\mathrm{OD}_{600}$ 1.8-2.0), mid-stationary (MS, $\mathrm{OD}_{600}$ 2.4-2.6), and late stationary phases (LS, grown $14-16 \mathrm{~h}$ after mid-stationary phase), and $\beta$-galactosidase activity was assayed. Consistent with previous reports (Perry et al., 2004; Sun Y.C. et al., 2012), hmsT::lacZ transcription and $h m s P::$ lac $Z$ transcription were only slightly affected by growth phase (Figures $3 A, B$ ). $h m s T:: l a c Z$ transcription was slightly lowered in the stationary phase (Figure 3A), while $h m s P:: l a c Z$ transcription was slightly increased in the stationary phase (Figure 3B). hmsC::lacZ transcription was higher with bacterial growth and especially was significantly higher in the late stationary phase (Figure 3C).

Next, we examined HmsT, HmsC, HmsD, HmsE, and HmsP expression under different growth phases by performing Western blotting. $3 \times$ Flag tags were added to the C-termini of HmsT, HmsP, and HmsC. $2 \times$ Myc tags were added to the C-terminus of $\mathrm{HmsD}$, and $3 \times \mathrm{HA}$ was added to the C-terminus of HmsE. Addition of these tags does not affect the function of these proteins (Ren et al., 2014; Guo et al., 2015 and data not shown). Each fusion protein was expressed under its own promoter on the chromosome at its native locus. HmsT-Flag and HmsP-Flag were constructed in the same strain; thus, the protein levels of $\mathrm{HmsT}$ and $\mathrm{HmsP}$ were detected at the same time using an anti-Flag antibody Supplementary Figure S2). Consistent with the results of lacZ reporter assay, the expressions of HmsT and HmsP were only slightly affected by growth phase, and HmsT expression was highest at early exponential phase, while HmsP expression was lowest at early exponential phase (Figure 3D). In addition, $\mathrm{HmsC}$ and $\mathrm{HmsD}$ were markedly increased in the late stationary phase (Figures $3 \mathrm{E}, \mathbf{F}$ ), which is consistent with the lacZ reporter assay (Figure 3C). However, HmsE expression was nearly undetectable in the late stationary phase (Figure 3G), suggesting that it is subject to post-transcriptional regulation. The transcriptional and protein levels of $h m s T, h m s P$, and $h m s C D E$ were almost constant at the early stationary phase; therefore we collected bacteria in this phase and analyzed the expression of these genes in the following experiment.

\section{The Effects of Environmental Signals on Expression of HmsT, HmsP, and HmsCDE}

Yersinia pestis biofilm formation and the intracellular c-di-GMP level were affected by environmental conditions. This might be because DGCs (HmsT and $\mathrm{HmsD}$ ) and PDE (HmsP) are differentially regulated in different environments. To verify this, we first examined the effect of environmental conditions on 


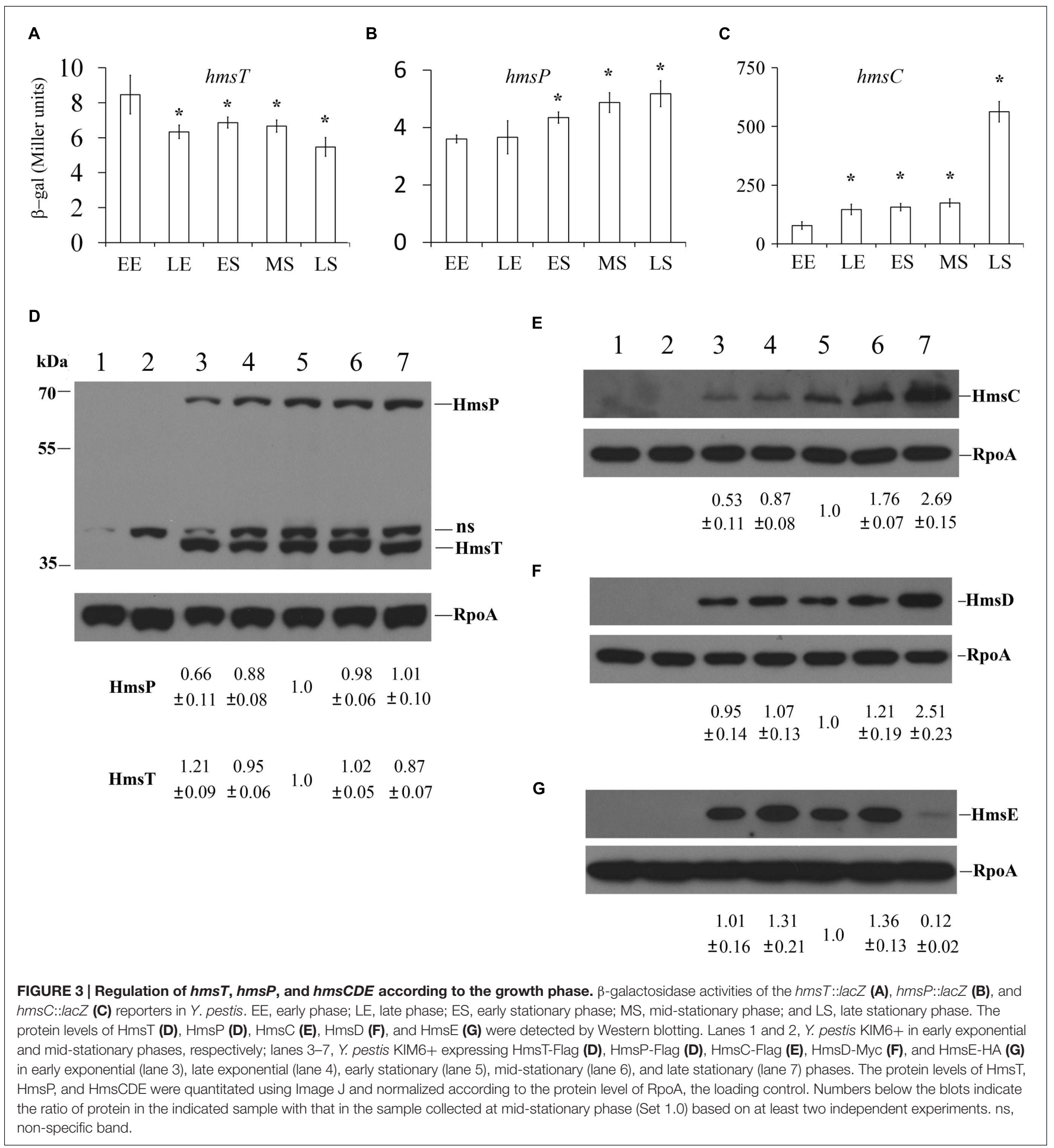

HmsT expression. We monitored the transcriptional level of $h m s T$ using the lac $Z$ reporter and the HmsT protein level by performing Western blotting. $h m s T$ transcription was increased by $\mathrm{CaCl}_{2}$, and a high temperature $\left(37^{\circ} \mathrm{C}\right)$ but was decreased by a low temperature $\left(21^{\circ} \mathrm{C}\right.$; Figure 4A). Consistent with the results of lacZ reporter assay, HmsT expression was not markedly affected (difference of less than $20 \%$ ) by most of the environmental signals tested, but was 1.38 -fold higher than in control cells after addition of $\mathrm{CaCl}_{2}$. The protein level of HmsT was increased at a low temperature (1.41-fold), and decreased at a high temperature (0.52-fold). This result is consistent with the previous reports that HmsT expression is post-transcriptionally regulated by temperature changes (Perry et al., 2004; Zhu et al., 2016). In addition, $\mathrm{NaCl}$ affected differentially the transcriptional levels 


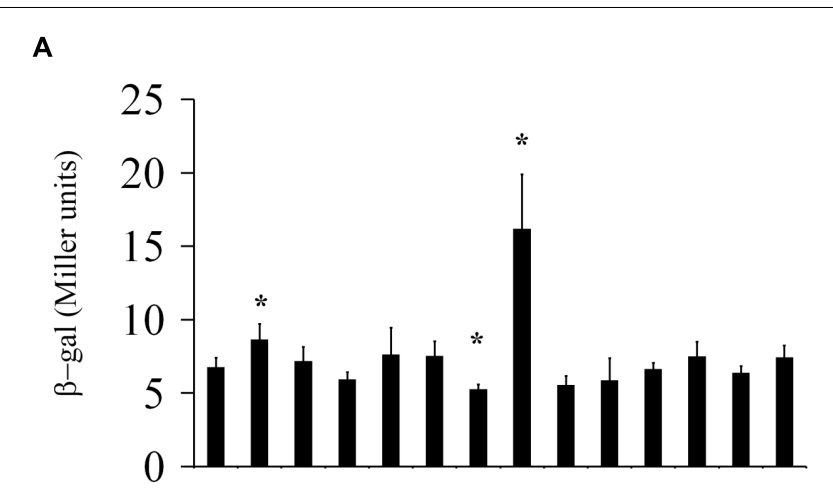

B

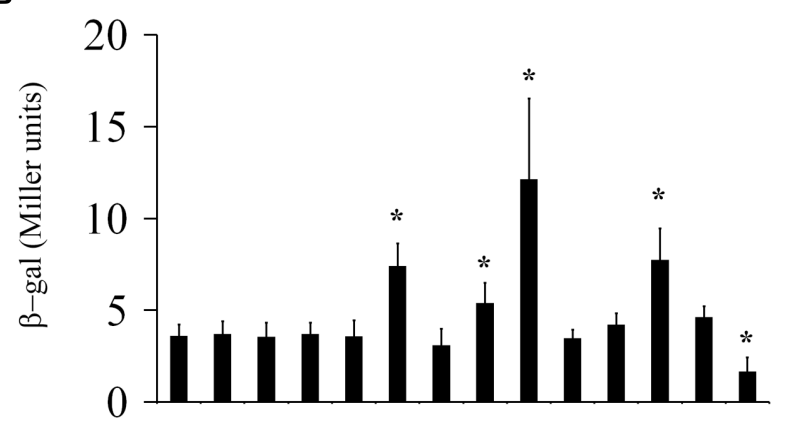

C

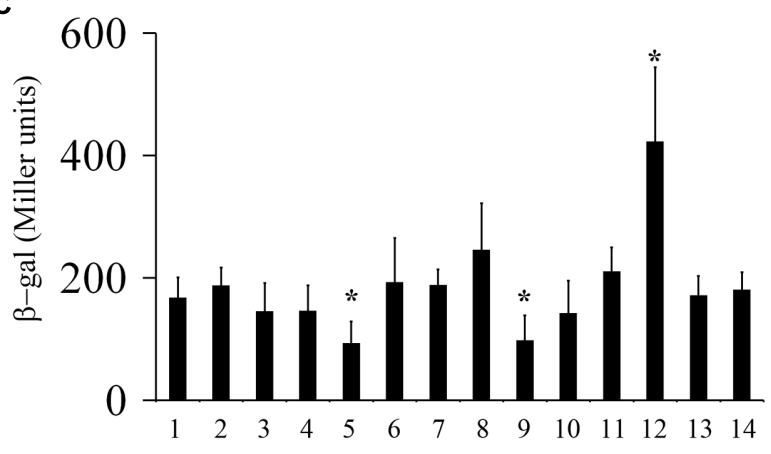

FIGURE 4 | Transcriptional regulation of $h m s T, h m s P$, and $h m s C D E$ by environmental signals. $\beta$-galactosidase activities of the $h m s T:: / a c Z$ (A), $h m s P:: / a c Z$ (B), and $h m s C:: / a c Z$ (C) reporters in Y. pestis grown in LB medium at $26^{\circ} \mathrm{C}(1), 21^{\circ} \mathrm{C}(7)$, or $37^{\circ} \mathrm{C}(8)$, or in $\mathrm{LB}$ medium supplemented with $\mathrm{CaCl}_{2}$ (2), $\mathrm{MgCl}_{2}$ (3), $\mathrm{CuSO}_{4}$ (4), $\mathrm{FeCl}_{2}(5), \mathrm{NaCl}$ (9), sucrose (10), $\mathrm{H}_{2} \mathrm{O}_{2}$ (11), DTT (12), DIP (13), or SDS (14), or at pH 5 at $26^{\circ} \mathrm{C} .{ }^{*} P<0.05$. The mean and standard deviation of three independent experiments are indicated.

and the protein levels of HmsT (Figures 4A and 5A), suggesting that Hms T expression is also post-transcriptionally regulated by salinity stress.

Next, we examined the effect of environmental conditions on HmsP expression using the lacZ reporter and Western blotting. Transcription of $h m s P$ was increased by a high concentration of $\mathrm{NaCl}$, a high temperature $\left(37^{\circ} \mathrm{C}\right)$, and an acid ( $\mathrm{pH}$ 5) environment, but was decreased with addition of SDS (Figure 4B). Consistent with the lacZ reporter assay, HmsP expression was not markedly affected (difference of less than
$30 \%$ ) by most of the tested environmental signals (Figure 5A), but was decreased in the presence of SDS (0.15-fold). In addition, although transcription of $h m s P$ was increased in the presence of a high concentration of $\mathrm{NaCl}$, and in acidic and redox (addition of DTT) environments (Figure 4B), the protein levels of HmsP were not markedly affected in these environmental conditions (Figure 5A), suggesting that Hms Pexpression is posttranscriptionally regulated by these environments.

Finally, we determined the effects of environment conditions on HmsCDE expression. We first determined the promoter activity of $h m s C$ using the $h m s C:: l a c Z$ reporter. The promoter activity of $h m s C$ was significantly decreased by high concentrations of $\mathrm{FeCl}_{2}$ or $\mathrm{NaCl}$, but was increased by a redox environment (Figure 4C). We further detected the protein level of HmsD by performing Western blotting. Consistent with the results of lac $Z$ reporter assay, the protein level of HmsD was not markedly affected (difference of less than 20\%) by most of tested environmental signals (Figure 5C). Expression of $\mathrm{HmsD}$ was increased in the redox (2.35-fold) environments, and decreased in the high concentration of $\mathrm{FeCl}_{2}$ (0.51-fold) and $\mathrm{NaCl}$ (0.53-fold; Figure 5C), which is consistent with the lacZ reporter assay (Figure $4 \mathrm{C}$ ). These results suggest that expression of $\mathrm{HmsD}$ is mainly regulated at the transcriptional level under these environmental conditions.

Because the activity of $\mathrm{HmsD}$ is regulated by $\mathrm{HmsC}$ and $\mathrm{HmsE}$ (Bobrov et al., 2014; Ren et al., 2014), we further determined the expression of $\mathrm{HmsC}$ and $\mathrm{HmsE}$ under various environmental conditions by performing Western blotting. By contrast to HmsD expression, which was not affected by most of the environmental conditions tested, the protein levels of $\mathrm{HmsC}$ and $\mathrm{HmsE}$ were markedly altered in the environmental conditions tested (Figures 5B,D). HmsC expression was increased by $\mathrm{CaCl}_{2}$ (1.31fold), $\mathrm{MgCl}_{2}$ (2.1-fold), $\mathrm{CuSO}_{4}$ (1.88-fold), and DIP (1.41-fold), and by a low temperature (1.98-fold), but was decreased by $\mathrm{FeCl}_{2}$ (0.28-fold), $\mathrm{NaCl}$ (0.09-fold), and sucrose (0.17-fold), and at $\mathrm{pH} 5$ (0.38-fold; Figure 5B). HmsE expression was markedly decreased by $\mathrm{CaCl}_{2}$ (0.67-fold), $\mathrm{NaCl}$ (0.57-fold), sucrose (0.18-fold), and SDS (0.48-fold) and at pH 5 (0.16-fold; Figure 5D). In addition, although neither the promoter activity of $h m s C$ (Figure $4 \mathrm{C}$ ) nor the $\mathrm{HmsD}$ protein level (Figure 5C) were significantly affected by the temperature, $\mathrm{HmsC}$ and $\mathrm{HmsE}$ expressions were markedly decreased by an increase in temperature (Figures 5B,D). These results suggest that the protein levels of $\mathrm{HmsC}$ and $\mathrm{HmsE}$ are more likely to be regulated than that of $\mathrm{HmsD}$ at the posttranscriptional level by environmental signals.

Taken together, these results suggest that HmsT, HmsP, and HmsCDE are differentially regulated by environmental signals, which might at least be partially responsible for changes in the intracellular c-di-GMP level and biofilm formation in Y. pestis in different environments.

\section{The Cumulative Effect of Environmental Signals on Y. pestis Biofilm Formation}

Biofilm formation can be affected by many environmental factors. $Y$. pestis forms a biofilm in the flea, however, little is known about the midgut physiology of the flea vector or environmental 
A


$\begin{array}{lllllll}0.92 & 1.0 & 0.72 & 0.85 & 1.03 & 0.99 & 1.18\end{array}$

$\begin{array}{rr}0.78 & 0.15 \\ \pm 0.09 & \pm 0.06\end{array}$

1.41

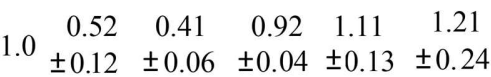

$\begin{array}{rrr}0.86 & 0.89\end{array}$

$\pm 0.12 \pm 0.06$

\section{B}

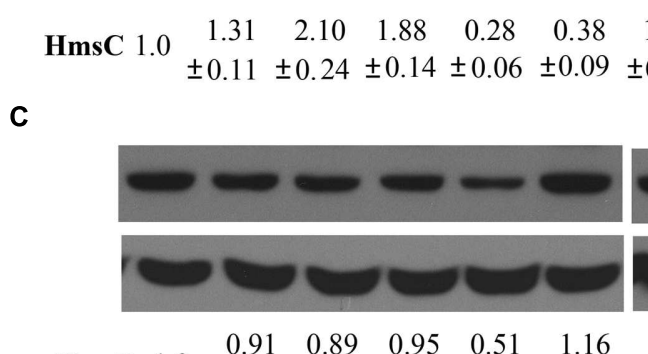

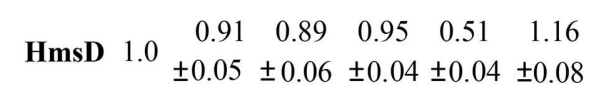
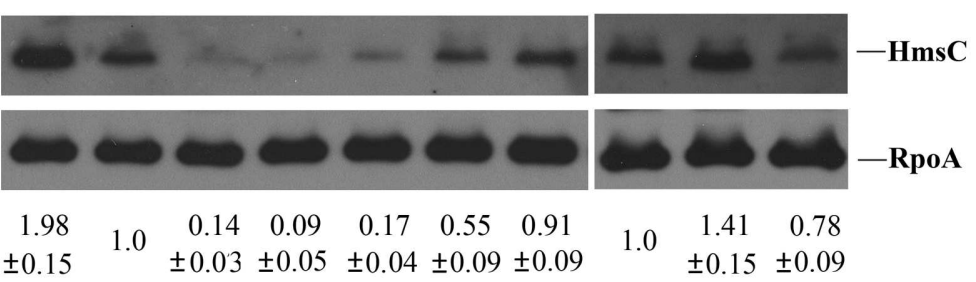

D

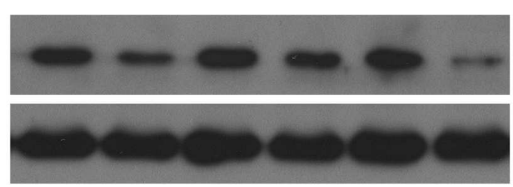

$\begin{array}{llllll}0.67 & 0.99 & 1.01 & 0.71 & 0.16 & 1.90\end{array}$

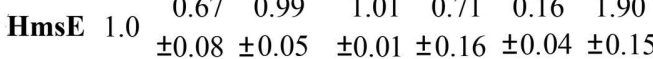

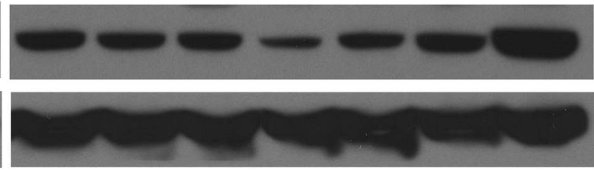

1.09

$\begin{array}{llllll}1.0 & 0.94 & 0.53 & 0.92 & 1.12 & 2.35\end{array}$

$\pm 0.06 \pm 0.07 \pm 0.02 \pm 0.10 \pm 0.34$
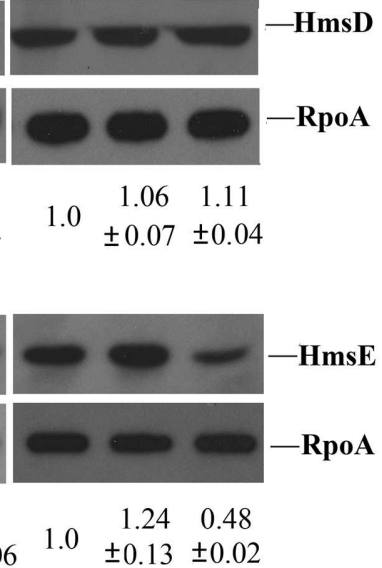

FIGURE 5 | Expression of Hmst, HmsP, and HmsCDE in various environmental conditions. (A) The protein levels of HmsT, HmsP. (B) The protein levels of HmsC. (C) The protein levels of HmsD. (D) The protein levels of HmsE. The total protein lysates used for detection were prepared from cells grown in LB medium at $26^{\circ} \mathrm{C}(1,8$, and 14$), 21^{\circ} \mathrm{C}(7)$, or $37^{\circ} \mathrm{C}$ (9), or in $\mathrm{LB}$ medium supplemented with $\mathrm{CaCl}_{2}$ (2), $\mathrm{MgCl}_{2}$ (3), $\mathrm{CuSO}_{4}$ (4), $\mathrm{FeCl}_{2}$ (5), $\mathrm{NaCl}$ (10), sucrose (11), $\mathrm{H}_{2} \mathrm{O}_{2}$ (12), DTT (13), DIP (15), or SDS (16), or at $\mathrm{pH} 5$ at $26^{\circ} \mathrm{C}$. The protein levels of $\mathrm{HmsT}$, HmsP, and $\mathrm{HmsCDE}$ were quantitated using Image $\mathrm{J}$ and normalized according to the protein level of RpoA, the loading control. Numbers below the blots indicate the ratio of protein in the indicated sample with that in the sample collected from cells grown in LB medium at $26^{\circ} \mathrm{C}$ based on at least two independent experiments. ns, non-specific band.

conditions in its digestive tract. The flea midgut $\mathrm{pH}$ is reportedly acidic (Perry and Fetherston, 1997), and probably between 6 and 7 (Zhou et al., 2012). Because HmsD plays a major role in the biofilm formation in acidic environment (Figure 1K), we believe that the acidic environment in the fleas might be one reason why $h m s D$ plays a central role in these insects (Sun et al., 2011).
A redox environment was another of the tested environmental conditions in which $h m s D$ played a major role in Y. pestis biofilm formation; therefore we analyzed the effect of a combination of an acidic and redox environment on biofilm formation. Upon addition of $1 \mathrm{mM}$ DTT, Y. pestis KIM6+ biofilm formation was decreased at various pHs (Figure 6A). 


\section{A}

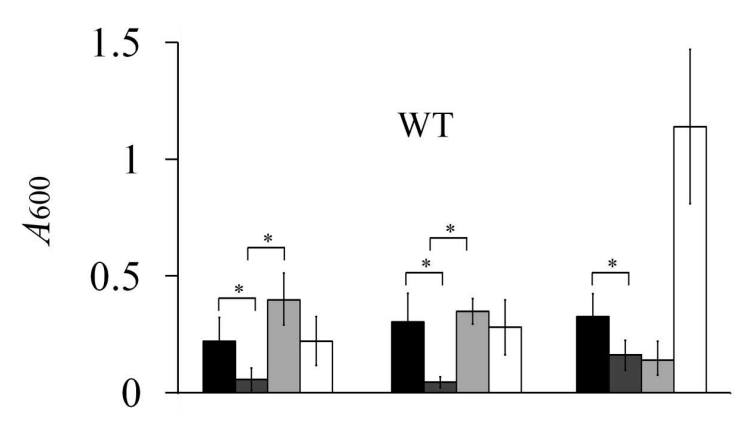

B

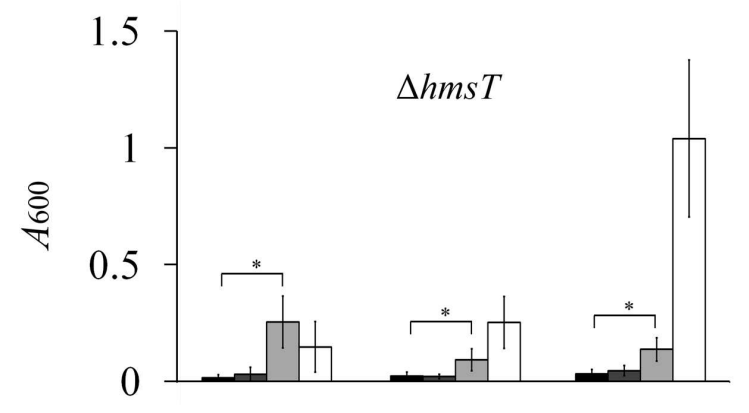

C

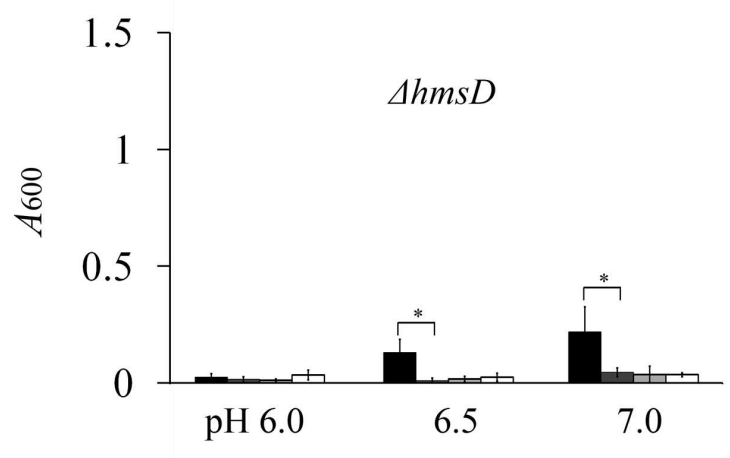

FIGURE 6 | The cumulative effect of environmental signals on Y. pestis biofilm formation. Relative amounts of adherent biofilm formed by the Y. pestis KIM6+ parental strain (A) and its isogenic derivatives, the $h m s T$ mutant (B) and the $h m s D$ mutant (C), in various environmental conditions. Black, dark gray, light gray, and white bars indicate cells were grown in medium supplemented with $0,1,2$, and $4 \mathrm{mM}$ DTT, respectively. The mean and standard deviation of three independent experiments are indicated. $* P<0.05$

By contrast, addition of $2 \mathrm{mM}$ DTT increased biofilm formation at $\mathrm{pH}$ 6.0, decreased formation at $\mathrm{pH}$ 7.0, and elicited nomarked change in formation at pH 6.5 (Figure 6A). Addition of $4 \mathrm{mM}$ DTT strongly increased biofilm formation at $\mathrm{pH} 7.0$, but did not significantly change biofilm formation at $\mathrm{pH} 6.0$ or 6.5 (Figure 6A). In the presence of DTT, biofilm formation was almost undetectable in the $h m s D$ mutant (Figure 6C). Biofilm formation in the $h m s T$ mutant was almost undetectable in the absence of DTT or the presence of $1 \mathrm{mM}$ DTT, but was significantly increased at $\mathrm{pH} 6-7$ in the presence of $2 \mathrm{mM}$ or $4 \mathrm{mM}$ DTT (Figure 6B). In addition, biofilm formation in the hms T mutant was decreased and increased by 2 and 4 mM DTT, respectively, as the $\mathrm{pH}$ increased from 6 to 7 (Figure 6B). Taken together these results suggest that various environmental signals can coordinately regulate $\mathrm{Hms}$, HmsP, and $\mathrm{HmsD}$ to modulate biofilm formation in $Y$. pestis.

Next, we analyzed the effects of combined environmental signals on HmsT, HmsP, and HmsCDE expression. HmsD expression was increased, whereas $\mathrm{HmsC}$ and $\mathrm{HmsE}$ expression was decreased by $2 \mathrm{mM}$ DTT in an acidic environment (Supplementary Figure S3), which might account for the increased biofilm formation of the hmsT mutant at these environments. Although, biofilm formation in the $h m s D$ mutant was decreased in the presence of $2 \mathrm{mM}$ DTT at pH 6 and 6.5 (Figure 6C), the protein levels of HmsT and HmsP were not significantly affected (Supplementary Figure S3), suggesting that the enzyme activities of HmsT and HmsP might be regulated by these environmental signals. Taken together these results further suggested that HmsT, HmsP, and HmsD respond to various environmental signals and function together to regulate biofilm formation.

\section{DISCUSSION}

Bacteria in nature are exposed to a constantly changing environment and their ability to sense and react to these changes is important for their survival. A characteristic feature of c-diGMP signaling networks is that a relatively large number of DGCs and PDEs contribute to changes in the intracellular c-diGMP concentration. For example, Vibrio cholera encodes 53, Pseudomonas aeruginosa encodes 38, and E. coli encodes 29 putative DGCs or PDEs predicted to modulate c-di-GMP levels, respectively (Galperin et al., 2001). These DGCs and PDEs might be differentially regulated in response to changes in the environment. Thus, it is challenging to understand how the c-diGMP level is controlled in these organisms. Y. pestis is an obligate pathogen that alternates between infection of mammals and fleas, and thus is only exposed to two different host environments. Thus, c-di-GMP signaling in Y.pestis is relatively simple and only three enzymes, two DGCs and one PDE, are involved in c-diGMP metabolism. Thus, Y. pestis is a good model to study the mechanism by which bacteria sense changes in the environment to regulate c-di-GMP signaling and biofilm formation.

In this study, we systematically determined how environmental changes regulate c-di-GMP signaling and biofilm formation in $Y$. pestis. We tested 12 environmental signals and found that biofilm formation in $Y$. pestis was markedly affected by addition of non-toxic concentrations of $\mathrm{CaCl}_{2}$, sucrose, $\mathrm{CuSO}_{4}, \mathrm{SDS}, \mathrm{FeCl}_{2}, \mathrm{NaCl}$, and DTT and by temperature changes (Figure 1). The biofilm phenotype was consistent with the cellular c-di-GMP level (Figure 2), suggesting environmental signals regulate biofilm formation mainly through regulation of the c-di-GMP level. In addition, our results suggested that DGCs and PDEs differentially respond to environmental changes and that the intracellular level of $\mathrm{c}$-di-GMP may reflect the cumulative 
activities of DGCs and PDEs. This is supported by the fact that addition of DTT and $\mathrm{pH}$ changes had different effects on biofilm formation in the wild-type, hms T mutant and hmsD mutant (Figures 1B,K and 6).

The cellular c-di-GMP level can be regulated by changes in the expression of c-di-GMP-metabolizing enzymes. The expression of genes encoding c-di-GMP metabolizing enzymes is affected by changes in environmental conditions. For example, transcription of genes encoding DGCs or PDEs in E. coli and $V$. cholerae decreases as the temperature increases (Sommerfeldt et al., 2009; Townsley and Yildiz, 2015). Here, we showed that the expression of HmsT, HmsP, and HmsD was differentially regulated by changes in environmental conditions at the transcriptional and post-transcriptional levels. Environmental changes changed the expression of HmsT and HmsP mainly at the post-transcriptional level. Although transcription of $h m s T$ was increased by high temperature (Figure 4A), its protein level of HmsT was decreased (Figure 5A). We have reported that the 3' UTR negatively regulates $h m s T$ mRNA stability in response to temperature change. In addition, Hms T protein might be degraded by proteases at high temperature (Perry et al., 2004). The protein level of HmsT was increased by addition of DTT and decreased by addition of $\mathrm{NaCl}$ (Figure 5A), whereas the transcription of $h m s T$ was unaffected by these environmental changes (Figure 4A). In addition, the transcription level of $h m s P$ was increased by acidic $(\mathrm{pH} 5)$ and redox (addition of DTT) environments, by high temperature $\left(37^{\circ} \mathrm{C}\right)$, and by a high concentration of $\mathrm{NaCl}$ (Figure 4B), but the protein level of HmsP was not markedly affected by these environmental changes (Figure 5A). The protein level of $\mathrm{HmsD}$ corresponded well with its transcriptional level (Figures 4C and 5C), suggesting environmental changes regulate the expression of $\mathrm{HmsD}$ mainly at the transcriptional level. The expression the c-di-GMP genes is probably regulated at the transcriptional level, posttranscriptional level (mRNA degradation), translational level and post-transcriptional level (protein degradation), and together these regulations probably serve to controland adapt the levels of these enzymes and c-di-GMP level to changes in the environment.

The cellular c-di-GMP level can also be affected by changes in the activities of c-di-GMP-metabolizing enzymes. The activities of DGCs in $V$. cholerae are differentially regulated at low temperatures. It was suggested that the sensory input domains of these DGCs play an important role in the sensing low temperatures. The activity of $\mathrm{HmsD}$ is suggested to be reciprocally regulated by $\mathrm{HmsC}$ and $\mathrm{HmsE}$. $h m s C$, $h m s D$, and $h m s E$ are co-transcribed from the same $h m s C$ promoter (Ren et al., 2014); however, their expression at the protein level differs depending on the environmental condition (Figure 5). The HmsD protein level was only moderately regulated by addition of DTT, $\mathrm{FeCl}_{2}$, or $\mathrm{NaCl}$ (Figure 5C), but $\mathrm{HmsC}$ and HmsE expression was moderately or strongly affected by most of environmental changes (Figures 5B,D). There are two possible explanations for this: (1) translation of $\mathrm{HmsC}$ and $\mathrm{HmsE}$ is particularly sensitive to environmental changes; and (2) more likely, the stabilities of $\mathrm{HmsC}$, a periplasmic protein, and $\mathrm{HmsE}$, an outer membrane protein, are particularly sensitive to environmental changes. Thus the c-di-GMP level and biofilm formation might quickly respond to the environmental changes through the regulation of $\mathrm{HmsC}$ and $\mathrm{HmsE}$ expression. For example, expression of $\mathrm{HmsD}$, HmsT and $\mathrm{HmsP}$ was not markedly affected by changes in $\mathrm{pH}$ (Figures 5A,C), whereas expression of $\mathrm{HmsC}$ and $\mathrm{HmsE}$ was markedly decreased by an acidic environment (Figures 5B,D), which might account for the increased biofilm formation in the hms T mutant in an acid environment. In addition, it has been reported that HmsE is important for the biofilm formation in vivo and in vitro (Bobrov et al., 2014), however, we found that HmsE does not play a significant role in the biofilm formation in Y. pestis in vitro (Ren et al., 2014). The medium that we used for the biofilm assay contained $4 \mathrm{mM} \mathrm{CaCl}_{2}$ and $4 \mathrm{mM} \mathrm{MgCl}_{2}$. A high concentration of $\mathrm{CaCl}_{2}$ or $\mathrm{MgCl}_{2}$ caused increased expression of $\mathrm{HmsC}$ but decreased expression of $\mathrm{HmsE}$ (Figures 5B,D), which might explain the different roles proposed for HmsE in previous reports.

HmsD plays a dominant role in $Y$. pestis biofilm formation in fleas, indicating that environmental conditions in the flea gut activate HmsD. Little is known about gut physiology and environmental conditions in the digestive tract of the flea. It has been suggested that the $\mathrm{Mg}^{2+}$ and $\mathrm{Ca}^{2+}$ levels in the flea midgut after consumption of blood meal are $0.8-1.0 \mathrm{mM}$ and 2.0-2.5 mM, respectively (Rebeil et al., 2013). In addition, the $\mathrm{pH}$ in the flea midgut is reportedly probably acidic (Perry and Fetherston, 1997). Other basic parameters such as osmotic pressure and redox potential in the flea gut are unknown. The acidic environment might be one reason why $h m s D$ plays a major role in controlling biofilm formation in the flea. This is supported by the fact that biofilm formation in $h m s T$ and $h m s D$ mutants was increased and decreased by an acidic environment, respectively (Figure 1K). There might be other unknown environmental signals that function together with an acidic environment to regulate the c-di-GMP signaling and biofilm formation in the flea gut. For example, a combination of an acidic environment and redox environment strongly activated $\mathrm{HmsD}$-dependent biofilm formation but repressed HmsT dependent biofilm formation (Figure 6).

Using $Y$. pestis as a model strain, we showed that multiple environmental changes modulate c-di-GMP signaling and biofilm formation. The expression or activity of c-di-GMPmetabolizing enzymes in $Y$. pestis is differentially regulated in response to these environmental changes, resulting in alternation of the intracellular c-di-GMP level, which in turn modulates biofilm formation and allows $Y$. pestis to adapt to changing environments. This study may be helpful to understand the c-diGMP signaling regulation in $Y$. pestis and other bacteria. The environmental conditions in the flea gut have not been fully investigated; thus, how $Y$. pestis regulates c-di-GMP signaling and biofilm formation to colonize fleas remains to be determined.

\section{MATERIALS AND METHODS}

\section{Bacterial Strains and Plasmids}

The strains and plasmids used are shown in Supplementary Table S1. The Y. pestis KIM6+ (Deng et al., 2002), cured 
of the $\mathrm{pCD} 1 / \mathrm{pYV}$ plasmid required for mammalian virulence, was used as the wild-type strain. $Y$. pestis mutants were made by a method to insert PCR products into the chromosome using the Red recombination system (Datsenko and Wanner, 2000; Sun et al., 2008). For strains containing reporter constructs hmsC::lacZ, hmsT::lacZ and $h m s:: l a c Z$, were constructed on the chromosome using a modification of the Red method (Gerlach et al., 2007; Sun Y.C. et al., 2012). The primers used for construction of the above strains and plasmids are listed in Supplemental Table S2. All strains and plasmids were verified by PCR, DNA sequencing and plasmid complementation, as appropriate.

\section{In Vitro Biofilm Assays}

Microtiter plate biofilm assays were performed as previously described with minor modifications (Ren et al., 2014). To determine the effects of $\mathrm{Ca}^{2+}$ and $\mathrm{Mg}^{2+}$, cells were grown in LB broth supplemented with appropriate concentration of $\mathrm{MgCl}_{2}$ or $\mathrm{CaCl}_{2}$ for $16-18 \mathrm{~h}$ at $26^{\circ} \mathrm{C}$, and diluted to $\mathrm{OD}_{600}$ of 0.05 using the same media. To determine the effect of temperature, cells were grown in $\mathrm{LB}$ broth supplemented with $4 \mathrm{mM} \mathrm{MgCl}$ and $4 \mathrm{mM} \mathrm{CaCl}_{2} 16-18 \mathrm{~h}$ at various temperature, diluted to $\mathrm{OD}_{600}$ of 0.05 using the same media. To determine the roles of other environmental conditions, cells were grown in LB broth supplemented with appropriate concentration of $4 \mathrm{mM} \mathrm{MgCl}$ and $4 \mathrm{mM} \mathrm{CaCl}_{2} 16-18 \mathrm{~h}$ at 26 , diluted to $\mathrm{OD}_{600}$ of 0.05 using the above media supplemented with appropriate concentrations of $\mathrm{CuSO}_{4}, \mathrm{FeCl}_{2}, \mathrm{DIP}, \mathrm{NaCl}$, sucrose, $\mathrm{H}_{2} \mathrm{O}_{2}, \mathrm{SDS}$, or DTT, or modified to a different $\mathrm{pH}$. Thereafter, $100 \mu \mathrm{L}$ resuspended cells were aliquoted into 96 -well polystyrene plates. The plates were incubated with shaking at $200 \mathrm{rpm}$ for $24 \mathrm{~h}$ at $26^{\circ} \mathrm{C}$ or an appropriate temperature. $\mathrm{OD}_{600}$ was measured and then the plates were washed three times with distilled water. The adherent biofilm was stained with $0.01 \%$ crystal violet for $15 \mathrm{~min}$. The dye was later re-dissolved with $80 \%$ ethanol and $20 \%$ acetone before the $A_{600}$ was measured. Results from three independent experiments with at least three replicates per experiment were analyzed by a one-way analysis of variance (ANOVA) with Dunnett's post-test.

\section{$\beta$-galactosidase Assays}

$\beta$-galactosidase activities were measured as previously described (Sun Y.C. et al., 2012). Overnight cultures of Y. pestis harboring appropriate lac $Z$ reporters were diluted with $50 \mathrm{~mL}$ of $\mathrm{LB}$ broth supplemented with $4 \mathrm{mM} \mathrm{CaCl} 2,4 \mathrm{mM} \mathrm{MgCl}_{2}, 2 \mathrm{mM} \mathrm{FeCl}_{2}$, $100 \mu \mathrm{M}$ DIP, $1 \mathrm{mM} \mathrm{CuSO}_{4}, 0.01 \%$ SDS, 6\% sucrose, $4 \mathrm{mM}$ DTT, $10 \mathrm{mM} \mathrm{H}_{2} \mathrm{O}_{2}$, or $4 \% \mathrm{NaCl}$, or modified to $\mathrm{pH} 5$, and grown at an appropriate temperature to the appropriate growth phase. ONPG (o-nitrophenyl- $b$-D-galactopyranoside) was cleaved by cell lysates at $37^{\circ} \mathrm{C}$ and this was in Miller units.

\section{Western Blotting}

Western blotting was performed as previously described (Ren et al., 2014). Overnight cultures of $Y$. pestis were diluted 1:1000 in $50 \mathrm{~mL}$ LB broth supplemented with $4 \mathrm{mM} \mathrm{CaCl}_{2}, 4 \mathrm{mM}$ $\mathrm{MgCl}_{2}, 2 \mathrm{mM} \mathrm{FeCl}$, $100 \mu \mathrm{M}$ DIP, $1 \mathrm{mM} \mathrm{CuSO} 4,0.01 \%$ SDS,
$6 \%$ sucrose, $4 \mathrm{mM}$ DTT, $10 \mathrm{mM} \mathrm{H}_{2} \mathrm{O}_{2}$, or $4 \% \mathrm{NaCl}$, or modified to different $\mathrm{pH} 5$ and grown at an appropriate temperature to the appropriate growth phase. Cells were collected and lysed by sonication. An appropriate amount of total proteins (100 ng total protein to detect $\mathrm{HmsD}$ and $\mathrm{HmsE}, 20 \mathrm{ng}$ total protein to detect $\mathrm{HmsC}$, HmsP, and $\mathrm{HmsT}$ ) was loaded and separated on 10 or $15 \%$ SDS-PAGE gels, transferred to PVDF membrane (Millipore), analyzed by immunoblotting with antibodies against HA (Sigma), Flag (Invitrogen), Myc (Invitrogen) or antiserum against RpoA, and detected with Immobilon Western HRP Substrate (Millipore). Results were quantitated by densitometry using NIH ImageJ and normalized according to the loading amount of RpoA.

\section{Measurement of c-di-GMP Levels}

Intracellular c-di-GMP levels in $Y$. pestis were detected as previously described (Ren et al., 2014). Overnight cultures of Y. pestis strains KIM6+ were diluted to an OD600 of 0.05 in $\mathrm{LB}$ broth supplemented with $4 \mathrm{mM} \mathrm{CaCl}_{2}, 4 \mathrm{mM} \mathrm{MgCl}$, $2 \mathrm{mM} \mathrm{FeCl}_{2}$, $100 \mu \mathrm{m}$ DIP, $1 \mathrm{mM} \mathrm{CuSO}$, 0.01\% SDS, 6\% sucrose, $4 \mathrm{mM}$ DTT, $10 \mathrm{mM} \mathrm{H}_{2} \mathrm{O}_{2}$, or $4 \% \mathrm{NaCl}$, or modified to $\mathrm{pH} 5$ and grown at an appropriate temperature to $\mathrm{OD}_{600}$ of 0.8 . Cell pellets were collected and resuspended in $50 \mu \mathrm{L}$ of extraction buffer (40\% methanol and 40\% acetonitrile prepared in $0.1 \mathrm{M}$ formic acid) per $48 \mathrm{mg}$ of wet cell weight. The slurries were incubated for $30 \mathrm{~min}$ at $-20^{\circ} \mathrm{C}$, and insoluble material was removed by centrifugation at $4^{\circ} \mathrm{C}$. The supernatants were neutralized by adding $4 \mu \mathrm{L}$ of $15 \% \mathrm{NH}_{4} \mathrm{HCO}_{3}$ per $100 \mu \mathrm{L}$ of sample. Ten microliters of each sample was analyzed using liquid chromatography tandem mass spectrometry. Synthetic c-di-GMP (BIOLOG Life Science Institute, Bremen, Germany) was used as a standard. Results from three independent experiments were analyzed using a one-way ANOVA with Dunnett's test.

\section{AUTHOR CONTRIBUTIONS}

Experiment designation: G-XR, Y-CS; Experiment carry out: G-XR, SF, X-PG, SC; Manuscript writing: G-XR, Y-CS, SF; Manuscript review and modification: G-XR, SC, X-PG,Y-CS.

\section{FUNDING}

This work was funded by the National Natural Science Foundation of China [81501723]; the National Basic Research Program of China (973 Program) [2015CB554200]; the National Science and Technology Major Project of China [2013ZX10004601]; and the Program for Changjiang Scholars and Innovative Research Team in University [IRT13007].

\section{SUPPLEMENTARY MATERIAL}

The Supplementary Material for this article can be found online at: http://journal.frontiersin.org/article/10.3389/fmicb.2016. 00821 


\section{REFERENCES}

Bacot, A. W. (1915). LXXXI. Further notes on the mechanism of the transmission of plague by fleas. J. Hyg. (Lond.) 14(Suppl.), 774.3-776.3.

Bacot, A. W., and Martin, C. J. (1914). LXVII. Observations on the mechanism of the transmission of plague by fleas. J. Hyg. (Lond.) 13(Suppl.), 423-439.

Bellows, L. E., Koestler, B. J., Karaba, S. M., Waters, C. M., and Lathem, W. W. (2012). Hfq-dependent, co-ordinate control of cyclic diguanylate synthesis and catabolism in the plague pathogen Yersinia pestis. Mol. Microbiol. 86, 661-674. doi: $10.1111 / \mathrm{mmi} .12011$

Bernier, S. P., Ha, D. G., Khan, W., Merritt, J. H., and O’Toole, G. A. (2011). Modulation of Pseudomonas aeruginosa surface-associated group behaviors by individual amino acids through c-di-GMP signaling. Res. Microbiol. 162, 680-688. doi: 10.1016/j.resmic.2011.04.014

Bobrov, A. G., Kirillina, O., Ryjenkov, D. A., Waters, C. M., Price, P. A., Fetherston, J. D., et al. (2011). Systematic analysis of cyclic di-GMP signalling enzymes and their role in biofilm formation and virulence in Yersinia pestis. Mol. Microbiol. 79, 533-551. doi: 10.1111/j.1365-2958.2010. 07470.x

Bobrov, A. G., Kirillina, O., Vadyvaloo, V., Koestler, B. J., Hinz, A. K., Mack, D., et al. (2014). The Yersinia pestis HmsCDE regulatory system is essential for blockage of the oriental rat flea (Xenopsylla cheopis), a classic plague vector. Environ. Microbiol. 17, 947-959. doi: 10.1111/1462-2920. 12419

Darby, C. (2008). Uniquely insidious: Yersinia pestis biofilms. Trends Microbiol. 16, 158-164. doi: 10.1016/j.tim.2008.01.005

Datsenko, K. A., and Wanner, B. L. (2000). One-step inactivation of chromosomal genes in Escherichia coli K-12 using PCR products. Proc. Natl. Acad. Sci. U.S.A. 97, 6640-6645. doi: 10.1073/pnas.120163297

Deng, W., Burland, V., Plunkett, G. III, Boutin, A., Mayhew, G. F., Liss, P., et al. (2002). Genome sequence of Yersinia pestis KIM. J. Bacteriol. 184, 4601-4611. doi: 10.1128/JB.184.16.4601-4611.2002

Galperin, M. Y., Nikolskaya, A. N., and Koonin, E. V. (2001). Novel domains of the prokaryotic two-component signal transduction systems. FEMS Microbiol. Lett. 203, 11-21. doi: 10.1111/j.1574-6968.2001. tb10814.x

Gerlach, R. G., Holzer, S. U., Jackel, D., and Hensel, M. (2007). Rapid engineering of bacterial reporter gene fusions by using Red recombination. Appl. Environ. Microbiol. 73, 4234-4242. doi: 10.1128/AEM. 00509-07

Guo, X. P., Ren, G. X., Zhu, H., Mao, X. J., and Sun, Y. C. (2015). Differential regulation of the hmsCDE operon in Yersinia pestis and Yersinia pseudotuberculosis by the Rcs phosphorelay system. Sci. Rep. 5:8412. doi: 10.1038/srep08412

Han, Y., Zhou, D., Pang, X., Zhang, L., Song, Y., Tong, Z., et al. (2005). Comparative transcriptome analysis of Yersinia pestis in response to hyperosmotic and high-salinity stress. Res. Microbiol. 156, 403-415. doi: 10.1016/j.resmic.2004. 10.004

Hengge, R. (2009). Principles of c-di-GMP signalling in bacteria. Nat. Rev. Microbiol. 7, 263-273. doi: 10.1038/nrmicro2109

Hinnebusch, B. J., and Erickson, D. L. (2008). Yersinia pestis biofilm in the flea vector and its role in the transmission of plague. Curr. Top. Microbiol. Immunol. 322, 229-248.

Hinnebusch, B. J., Perry, R. D., and Schwan, T. G. (1996). Role of the Yersinia pestis hemin storage (hms) locus in the transmission of plague by fleas. Science 273 , 367-370. doi: 10.1126/science.273.5273.367

Jarrett, C. O., Deak, E., Isherwood, K. E., Oyston, P. C., Fischer, E. R., Whitney, A. R., et al. (2004). Transmission of Yersinia pestis from an infectious biofilm in the flea vector. J. Infect. Dis. 190, 783-792. doi: 10.1086/ 422695

Kirillina, O., Fetherston, J. D., Bobrov, A. G., Abney, J., and Perry, R. D. (2004). HmsP, a putative phosphodiesterase, and HmsT, a putative diguanylate cyclase, control Hms-dependent biofilm formation in Yersinia pestis. Mol. Microbiol. 54, 75-88. doi: 10.1111/j.1365-2958.2004.04253.x

Koestler, B. J., and Waters, C. M. (2013). Exploring environmental control of cyclic di-GMP signaling in Vibrio cholerae by using the ex vivo lysate cyclic di-GMP assay (TELCA). Appl. Environ. Microbiol. 79, 5233-5241. doi: 10.1128/AEM.01596-13

Koestler, B. J., and Waters, C. M. (2014). Bile acids and bicarbonate inversely regulate intracellular cyclic di-GMP in Vibrio cholerae. Infect. Immun. 82, 3002-3014. doi: 10.1128/IAI.01664-14

Malone, J. G., Jaeger, T., Manfredi, P., Dotsch, A., Blanka, A., Bos, R., et al. (2012). The YfiBNR signal transduction mechanism reveals novel targets for the evolution of persistent Pseudomonas aeruginosa in cystic fibrosis airways. PLoS Pathog. 8:e1002760. doi: 10.1371/journal.ppat. 1002760

Perry, R. D., Bobrov, A. G., Kirillina, O., Jones, H. A., Pedersen, L., Abney, J., et al. (2004). Temperature regulation of the hemin storage (Hms+) phenotype of Yersinia pestis is posttranscriptional. J. Bacteriol. 186, 1638-1647. doi: 10.1128/JB.186.6.1638-1647.2004

Perry, R. D., and Fetherston, J. D. (1997). Yersinia pestis-etiologic agent of plague. Clin. Microbiol. Rev. 10, 35-66.

Rebeil, R., Jarrett, C. O., Driver, J. D., Ernst, R. K., Oyston, P. C., and Hinnebusch, B. J. (2013). Induction of the Yersinia pestis PhoP-PhoQ regulatory system in the flea and its role in producing a transmissible infection. J. Bacteriol. 195, 1920-1930. doi: 10.1128/JB.02000-12

Rempe, K. A., Hinz, A. K., and Vadyvaloo, V. (2012). Hfq regulates biofilm gut blockage that facilitates flea-borne transmission of Yersinia pestis. J. Bacteriol. 194, 2036-2040. doi: 10.1128/JB.06568-11

Ren, G. X., Yan, H. Q., Zhu, H., Guo, X. P., and Sun, Y. C. (2014). HmsC, a periplasmic protein, controls biofilm formation via repression of $\mathrm{HmsD}$, a diguanylate cyclase in Yersinia pestis. Environ. Microbiol. 16, 1202-1216. doi: $10.1111 / 1462-2920.12323$

Romling, U. (2012). Cyclic di-GMP, an established secondary messenger still speeding up. Environ. Microbiol. 14, 1817-1829. doi: 10.1111/j.14622920.2011.02617.x

Romling, U., Galperin, M. Y., and Gomelsky, M. (2013). Cyclic di-GMP: the first 25 years of a universal bacterial second messenger. Microbiol. Mol. Biol. Rev. 77, 1-52. doi: 10.1128/MMBR.00043-12

Ryan, R. P., Fouhy, Y., Lucey, J. F., Crossman, L. C., Spiro, S., He, Y. W., et al. (2006). Cell-cell signaling in Xanthomonas campestris involves an HD-GYP domain protein that functions in cyclic di-GMP turnover. Proc. Natl. Acad. Sci. U.S.A. 103, 6712-6717. doi: 10.1073/pnas.06003 45103

Ryjenkov, D. A., Tarutina, M., Moskvin, O. V., and Gomelsky, M. (2005). Cyclic diguanylate is a ubiquitous signaling molecule in bacteria: insights into biochemistry of the GGDEF protein domain. J. Bacteriol. 187, 1792-1798. doi: 10.1128/JB.187.5.1792-1798.2005

Schmidt, A. J., Ryjenkov, D. A., and Gomelsky, M. (2005). The ubiquitous protein domain EAL is a cyclic diguanylate-specific phosphodiesterase: enzymatically active and inactive EAL domains. J. Bacteriol. 187, 4774-4781. doi: 10.1128/JB.187.14.4774-4781.2005

Simm, R., Fetherston, J. D., Kader, A., Romling, U., and Perry, R. D. (2005). Phenotypic convergence mediated by GGDEF-domain-containing proteins. J. Bacteriol. 187, 6816-6823. doi: 10.1128/JB.187.19.68166823.2005

Sommerfeldt, N., Possling, A., Becker, G., Pesavento, C., Tschowri, N., and Hengge, R. (2009). Gene expression patterns and differential input into curli fimbriae regulation of all GGDEF/EAL domain proteins in Escherichia coli. Microbiology 155(Pt 4), 1318-1331. doi: 10.1099/mic.0. 024257-0

Sun, F., Gao, H., Zhang, Y., Wang, L., Fang, N., Tan, Y., et al. (2012). Fur is a repressor of biofilm formation in Yersinia pestis. PLoS ONE 7:e52392. doi: 10.1371/journal.pone.0052392

Sun, Y. C., Guo, X. P., Hinnebusch, B. J., and Darby, C. (2012). The Yersinia pestis Rcs phosphorelay inhibits biofilm formation by repressing transcription of the diguanylate cyclase gene hmsT. J. Bacteriol. 194, 2020-2026. doi: 10.1128/JB.06243-11

Sun, Y. C., Hinnebusch, B. J., and Darby, C. (2008). Experimental evidence for negative selection in the evolution of a Yersinia pestis pseudogene. Proc. Natl. Acad. Sci. U.S.A. 105, 8097-8101. doi: 10.1073/pnas.0803 525105

Sun, Y. C., Koumoutsi, A., Jarrett, C., Lawrence, K., Gherardini, F. C., Darby, C., et al. (2011). Differential control of Yersinia pestis biofilm formation in vitro and 
in the flea vector by two c-di-GMP diguanylate cyclases. PLoS ONE 6:e19267. doi: 10.1371/journal.pone.0019267

Townsley, L., and Yildiz, F. H. (2015). Temperature affects c-di-GMP signalling and biofilm formation in Vibrio cholerae. Environ. Microbiol. 17, 4290-4305. doi: 10.1111/1462-2920.12799

Zhou, J., Sayre, D. A., Wang, J., Pahadi, N., and Sintim, H. O. (2012). Endo-S-cdi-GMP analogues-polymorphism and binding studies with class I riboswitch. Molecules 17, 13376-13389. doi: 10.3390/molecules171113376

Zhu, H., Mao, X. J., Guo, X. P., and Sun, Y. C. (2016). The hmsT 3' untranslated region mediates c-di-GMP metabolism and biofilm formation in Yersinia pestis. Mol. Microbiol. 99, 1167-1178. doi: 10.1111/mmi.13301
Conflict of Interest Statement: The authors declare that the research was conducted in the absence of any commercial or financial relationships that could be construed as a potential conflict of interest.

Copyright (c) 2016 Ren, Fan, Guo, Chen and Sun. This is an open-access article distributed under the terms of the Creative Commons Attribution License (CC BY). The use, distribution or reproduction in other forums is permitted, provided the original author(s) or licensor are credited and that the original publication in this journal is cited, in accordance with accepted academic practice. No use, distribution or reproduction is permitted which does not comply with these terms. 